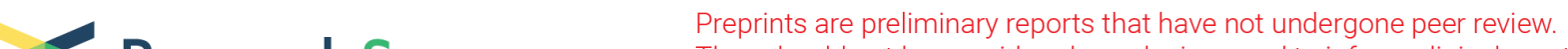 $\begin{array}{ll}\text { Research Square } & \text { They should not be considered conclusive, used to inform clinical practice, } \\ \text { or referenced by the media as validated information. }\end{array}$
}

\section{Retrospective Analysis of the Clinical and Image Presentation of Eight Primary Benign Mediastinal Schwannomas}

\section{Ramiro Sandoval-Macias}

IMSS: Instituto Mexicano del Seguro Social

Irving Daniel Ortiz-Sanchez

IMSS: Instituto Mexicano del Seguro Social

Ana Lilia Remirez-Castellanos

IMSS: Instituto Mexicano del Seguro Social

Luis Mora Hernandez

IMSS: Instituto Mexicano del Seguro Social

Candelaria Cordova Uscanga

IMSS: Instituto Mexicano del Seguro Social

Alejandra Mantilla Morales

IMSS: Instituto Mexicano del Seguro Social

\section{Tania Alejandra Galindo-García}

Instituto Nacional de Ciencias Medicas y Nutricion Salvador Zubiran

\section{Armando Gamboa-Dominguez}

Instituto Nacional de Ciencias Medicas y Nutricion Salvador Zubiran

Fernando Candanedo-Gonzalez ( $\nabla$ fa_candanedo@yahoo.com.mx )

Instituto Nacional de Ciencias Medicas y Nutricion Salvador Zubiran https://orcid.org/0000-00026926-8182

\section{Research note}

Keywords: Schwannoma, Mediastinum, Computed tomography, Histological analysis, Immunohistochemistry, Neurogenic markers, Treatment, Follow-up

Posted Date: March 19th, 2021

DOI: https://doi.org/10.21203/rs.3.rs-322305/v1

License: (c) (i) This work is licensed under a Creative Commons Attribution 4.0 International License. Read Full License 
Version of Record: A version of this preprint was published at BMC Research Notes on July 21st, 2021. See the published version at https://doi.org/10.1186/s13104-021-05694-6. 


\section{Abstract}

Objective: Mediastinal schwannomas sometimes can be confused with other neoplasms in the initial radiological studies, especially when there is a history of cancer in another site and that require a more accurate analysis by computed tomography (CT) or even magnetic resonance (MRI). Our study was aimed to perform a retrospective analysis of the clinical and imaging features in a series of patients with mediastinal schwannomas that were confirmed by histology and immunohistochemistry.

Results: We found eight patients, five man and three women with an average age of 51 years. The main signs and symptoms at time of diagnosis were chest pain, dyspnea, cough and dysphagia. CT showed that the tumor was located in the posterior compartment of the chest in $7 / 8$ cases. Tumors $>10 \mathrm{~cm}$ were more heterogeneous and showed cystic changes. All cases underwent posterolateral thoracotomy and radiological follow-up showed no evidence of recurrence. Histological analysis was the gold standard to confirm diagnosis in addition to at least one neurogenic IHC marker. In conclusion, mediastinal schwannomas are benign encapsulated tumors. By CT, schwannomas $>10 \mathrm{~cm}$ showed cystic degeneration more frequently. Posterolateral thoracotomy allows complete resection and is considered the surgical approach of choice.

\section{Introduction}

Schwannomas are benign neoplasms that arise from Schwann cells that surround peripheral nerve fibers. ${ }^{[1,2]}$ Less than $9 \%$ of schwannomas occur in the mediastinum. ${ }^{[3]}$ They often originate at the root of a spinal nerve and may involve the thoracic nerve. More than $90 \%$ of these lesions are unique and sporadic. ${ }^{[3]}$ They have a peak incidence between fourth to sixth decades of life. ${ }^{[3]}$ Even though there are case reports of giant mediastinal schwannomas, ${ }^{[4-6]}$ they generally measure less than $5 \mathrm{~cm}$ at the time of diagnosis, they are characterized by its slow growing, and lack of specific symptoms; therefore, a common presentation occurs as a well-defined lesion without a mass effect while performing a radiographic study aimed to various other complaints. However, when schwannomas are large, it is possible for them to present by localized pain; histologically, common features are cystic degeneration, low cellularity, hemorrhage and small calcifications. ${ }^{[3]}$ There are few studies in the Mexican population that analyze the characteristics of mediastinal schwannomas. ${ }^{[7,8]}$ Our objective was to perform a retrospective analysis of the clinical and tomographic features of patients with primary benign mediastinal schwannoma.

\section{Main Text}

\section{Material and methods}

\section{Study design and population characteristics}


A retrospective study was performed that included patients with a diagnosis of mediastinal schwannoma. All cases were obtained from the surgical pathology archives of UMAE Hospital de Oncologia Centro Medico Nacional Siglo XXI. IMSS, from January 2011 to January 2018. Permission was requested in the clinical file to review patient records. Clinical features such as age, gender, time of evolution, type of treatment and follow-up data obtained from medical records. Its association with neurofibromatosis type 2, schwanomatosis and Gorlin-Koutlas syndrome was also investigated. From the radiology file, chest CT scans from each patient was obtained and submitted for re-assessment by two experienced radiologists who investigated the location, shape, size and features of each lesion (ALRC and $\mathrm{LMH}$ ). All cases were reevaluated by three experienced pathologists (CCU, AMM and FCG) using the WHO criteria. ${ }^{[9]}$

\section{Immunohistochemistry}

All tissues were fixed in formaldehyde buffered at $10 \%$ and paraffin embedded. Histological sections were stained with hematoxylin and eosin. For immunohistochemistry (IHC) analysis, $5-\mu m$ sections of a representative block in each case were obtained. The following antibodies were used: S100 protein (Biosb), glial fibrillar acid protein (GFAP; Invitrogen), CD34 (Biocare), and Ki-67 (Biosb), which were performed on an automated immunostainer (Ventana, Biotek System Tucson, Ariz) with an appropriate positive and negative control run simultaneously. Briefly, paraffin sections mounted on charged glass slides, air -dried over-night, and then de-paraffinized. To enhance immunostaining, a heat-induced epitope-retrieval procedure was performed. After incubation with blocking serum, sections were incubated with primary antibodies, followed by a biotinylated polyvalent secondary antibody solution. All sections were then incubated with horseradish peroxidase-conjugated avidin-biotin complex, followed by 3,3diaminobenzidine and hydrogen peroxidase.

\section{Results}

\section{Clinical features}

During the period analyzed, 163 primary soft tissue tumors of the mediastinum were resected, of which only eight patients corresponded to mediastinal schwannomas (5\%). Five men (63\%) and three women (37\%). The average age was 51 years (range, 32-78 years). The average age of the women was 43 years versus 56 years for men. Seventy-five percent of the patients had symptoms. The main clinical manifestations were chest pain in four patients (64\%), dyspnea in one patient $(17 \%)$, cough in one patient $(17 \%)$, dysphagia in one patient $(17 \%)$ and two patients were asymptomatic $(25 \%)$. No weight loss found in any patient. The average time of clinical evolution was 4 months (range, 1 to 6 months). In none of our cases was found an association with neurofibromatosis type 2, schwannomatosis or Gorlin-Koutlas syndrome.

\section{Tomographic features}


All patients had a chest X-ray and a chest CT, which allowed identifying the lesion. Chest X-ray films showed a well-defined round radiopaque mass in all cases. Only in three cases, a diagnosis of schwannoma was suspected at the time of CT. In one patient, the mediastinal tumor was identified as an asymptomatic finding secondary to a kidney transplant check-up. Other of our patients had a history of clinical stage IIB breast cancer, which in follow-up with chest X-ray revealed a mediastinal mass in the left hemithorax. Initially it was thought that it could correspond to recurrence of the disease. This was ruled out by performing a CT scan in which the diagnosis of schwannoma was proposed, leading the patient to a surgical approach.

In seven patients, the tumor was located in the posterior mediastinum ( $88 \%$ ) by CT; in one of them, the mass was located in the posterior upper para-esophageal compartment. No patient had evidence of tumoral activity was observed elsewhere. In all cases, the mass was ovoid and well defined. In four cases, the lesion was homogeneous and in four cases it showed heterogeneous reinforcement with hypo and hyperintense areas after the application of contrast material (Fig. 1a and b).

\section{Pathologic findings}

Macroscopically, all cases were solitary tumors, with ovoid growth, encapsulated, with gray-yellow outer surface. The average size of the tumors was $8 \mathrm{~cm}$ (range, 3.5-14.0 cm). The average size of the tumors in the group of women was $8.6 \mathrm{~cm}$ (range, $4.0-14.0 \mathrm{~cm}$ ) versus $7.16 \mathrm{~cm}$ in the male gender (range, 3.5-11.0 $\mathrm{cm}$ ). Fifty percent of the tumors showed cystic degeneration. These tumors had an average size of 10.0 $\mathrm{cm}$ (range, 7.0-14.0 cm) compared to tumors without cystic changes which had an average size of $5.4 \mathrm{~cm}$ (range, 3.5-8, $0 \mathrm{~cm}$ ) (Fig. 1c and d). All cases were completely resected and in none was necrosis.

\section{Histopathological features}

Histologically, only in three cases (38\%), a small nerve fragment was identified in the extracapsular portion (Fig. 2a). Four cases showed cystic changes (50\%; Fig. 2b). Six cases presented myxoid areas $(75 \%)$ and in five cases there was hemorrhage (Fig. 2c). All cases showed hypercellular areas forming short bundles (Antoni A) alternating with scarce formation of palisades (bodies of Verocay) with hypocellular component (Antoni B) (Fig. 2d). The neoplastic cells were spindle-shaped, with twisted nuclei and indistinct cytoplasmic borders, immersed in a collagenized stroma. (Figure 2e). One case (case \# two) showed senescent changes with greater nuclear atypia and hyalinized stroma (Fig. 2f).

\section{Immunohistochemistry findings}

By IHC, all cases were intensely positive for $\mathrm{S} 100$ protein in nucleus and cytoplasm, and showed focal expression of GFAP, mainly in Antonia A areas. Neither case showed expression for CD34. The proliferation index with $\mathrm{Ki}-67$ was less than $1 \%$ in all cases. One cases (case \# five) showed hypercellularity with a storiform pattern in approximately $50 \%$ of the tumor mimicking solitary fibrous tumor. However, the neoplastic cells were positive for two neurogenic markers (S100 protein and GFAP) and negative for CD34 (Fig. 3). 


\section{Treatment and follow-up of patients}

All the patients underwent surgery through posterolateral thoracotomy and radiological follow-up. In four cases, the thoracotomy was on the left-sided versus three right-sided cases. In all patients the tumor resected completely. The mean follow-up of the patients was 27 months (range 0-48 months). All patients were found alive without clinical or radiological recurrence of the disease at their last visit. In none of the patients a malignant transformation of the disease was diagnosed.

\section{Discussion}

It is estimated that $9 \%$ of all schwannomas occur in the mediastinum. ${ }^{[10]}$ Fifty percent of neurogenic tumors of the mediastinum are schwannomas. ${ }^{[3]}$ In our series, schwannomas represented $5 \%$ of all soft tissue tumors of the mediastinum. More than $90 \%$ of schwannomas are solitary, sporadic, and slowgrowing neoplasms. These lesions affect both genders equally, all age groups reached a higher incidence between the fourth and sixth decades of life. ${ }^{[1-3]}$ In our series, the average age was 51 years while women were found to be a decade younger than men.

The clinical history, physical examination and image evaluation must be considered. Mediastinal schwannomas can originate from spinal, paravertebral sympathetic branches, vagus, phrenic and intercostal nerves, respectively. ${ }^{[1-3,11]}$ In our study, about half of cases originated in the paravertebral region. The classic presentation is an incidental asymptomatic mass found on routine investigations as simple as a chest X-ray. ${ }^{[12]}$ Interestingly, some patients experience paresthesia or chest pain from compression of adjacent structures or extensions of the tumor in the thoracic spine. Other clinical manifestations are dry cough, dyspnea, and dysphagia. In our study and in contrast to other international publications, $57 \%$ of the patients had symptoms attributable to a mediastinal schwannoma. The ideal treatment consists of video-assisted thoracic surgery with complete resection of the mass because of its subsequent growth and mass-effect. ${ }^{[1-3]}$ In our study, all patients underwent open posterolateral thoracotomy as standard.

CT and MRI can help accurately to determine the exact location of the mediastinal tumor and also defines its relationship to adjacent structures. On CT scanning, schwannomas appears as well-defined rounded mass with smooth margins, they are isodense or hypodense in the paravertebral region or long the courses of intercostal nerves. ${ }^{[13]}$ They have a variable enhancement pattern after administration of the intravenous contrast material including multiple hypodense or cystic areas with central hypodensity and central enhancement, as well as peripheral hypodensity. ${ }^{[13,14]}$ Calcifications are occasionally detected, and low attenuation correlates with areas of hypocellularity, cystic change, hemorrhage, and lipidisation. ${ }^{[3,15]}$

While there are few cases reports of giant mediastinal schwannomas, these are often identified incidentally by chest X-ray, followed by CT scans that show a clearly defined mass with low densities and mild enhancement. ${ }^{[4-6]}$ Giant schwannomas are more heterogeneous with cystic degeneration. ${ }^{[3]}$ In our 
series, cystic degeneration was observed more frequently in tumors larger than $10 \mathrm{~cm}$. Tumors smaller than $10 \mathrm{~cm}$ were more frequently homogeneous with solid areas in the absence of cystic degeneration. This supports the idea that degenerate changes directly related to the size of the tumor. Heterogeneous changes observed by CT have a histological translation. In our study, the schwannomas that were homogenous by CT more frequently identified in the histological analysis of classical areas Antoni $A$ and Antoni B. While in tumors that were heterogeneous by CT scan, they presented microscopically extensive hypocellular areas with marked cystic degeneration, reticular pattern, edema, hemorrhage and sometimes calcifications. However, by IHC positivity for $\mathrm{S} 100$ protein confirms the diagnosis in all cases.

Mediastinal schwannomas can be misdiagnosed as lymph node metastasis from locally advanced breast cancer. ${ }^{[16]}$ The clinical distinction between mediastinal schwannoma and metastasis in cases that have a history of cancer, can be difficult and requires its surgical removal and histopathological analysis. The location and composition through imaging studies are critical to make a differential diagnosis. Even through sophisticated studies such a ${ }^{18} \mathrm{~F}$-fluorodeoxyglucose positron emission tomography and computed tomography ( ${ }^{18} \mathrm{~F}-\mathrm{FDG}$ PET/CT), mediastinal schwannoma can simulate metastases to breast cancer lymph nodes, as shown by Martinez-Esteve et al. ${ }^{[16]}$ Similarly, we found a patient with a clinical history of stage IIB breast cancer during follow-up showed a mediastinal mass on a chest X-ray that was initially thought to be a recurrence of the disease. However, when a CT scan was performed, the site of origin of the tumor was defined precisely and it proposed that it could correspond to schwannoma. Finally, it was resected and the histopathological analysis showed a schwannoma with diffuse expression of S100 protein and GFAP.

Cellular schwannoma are an infrequent variant with a predominantly cellular growth pattern, in the absence of Verocay bodies. Usually seen at paravertebral, pelvic, retroperitoneal, or mediastinal location. [17] This variant can be confused with a malignant peripheral nerve sheath tumor or with a solitary fibrous tumor. ${ }^{[18,19]}$ In our study, we found only one case of a cellular schwannoma (13\%) with storiform pattern mimicking a solitary fibrous tumor. The diagnosis of schwannoma was supported by light microscopy and corroborated by S100 protein and GFAP expression and negative for CD34.

\section{Conclusion}

Mediastinal schwannomas are benign encapsulated tumors, which on CT are more frequently located in the posterior compartment. Tumors $>10 \mathrm{~cm}$ are usually heterogeneous with cystic degeneration. Posterolateral thoracotomy allows complete resection and is the surgical approach of choice. Histological analysis remains the gold standard to confirm the diagnosis along with IHC markers that include at least one neurogenic marker.

\section{Limitations}

It is a retrospective study with a small sample. Therefore, a large-scale study that includes a larger number of patients is necessary. 


\section{Abbreviations}

AFINES: Program to support and promote student research; CT: Computed tomography; ${ }^{18} \mathrm{~F}-\mathrm{FDG}$ PET/CT: ${ }^{18} \mathrm{~F}$-fluorodeoxyglucose positron emission tomography and computed tomography; GFAP: Glial fibrillary acid protein; IHC: Immunohistochemistry; MRI: Magnetic resonance imaging; UMAE: Unidad Medica de Alta Especialidad; UNAM: Universidad Nacional Autonoma de Mexico; X-ray: radiograph.

\section{Declarations}

\section{Acknowledgments}

We wish to thank Jose Antonio Zimmermann Mireles, MD, for her assistance in reviewing the English language. We wish to thank Ricardo Aguilar Guadarrama for her assistance in immunohistochemistry.

\section{Author's contribution}

RSM, IDOS, ALRC, LMH, CCU, AMM, AGD, and FCG have substantial contributions to conception and design of the study; RSM, IDOS and FCG collected the sample and analyzed the data, also made contributions to acquisition of clinical data; ALRC and LMH analyzed and describe the characteristics of the tumor by CT and MRI; CCU, AMM, AGD, and FCG evaluated the histological findings and the results of the IHC; AMM, AGD and FCG draft the paper. All authors have given approval the final version of manuscript to be published.

\section{Funding}

No funding has been gained by the authors for the research.

\section{Availability of data and materials}

Not applicable.

\section{Ethics approval and consent to participate}

The study was approved by the ethics committee of the UMAE Hospital de Oncologia Centro Médico Nacional Siglo XXI (R-2019-3001-013). In addition, informed consent was obtained from each of the patients.

\section{Consent to publish}

Not applicable.

\section{Competing of interest}

The authors declare that they no competing interest. 


\section{Author details}

${ }^{1}$ AFINES program. School of Medicine, Ciudad Universitaria. UNAM. ${ }^{2}$ Department of Radiology. UMAE Hospital de Oncologia, Centro Medico Nacional Siglo XXI. IMSS. ${ }^{3}$ Department of Pathology. UMAE Hospital de Oncologia, Centro Medico Nacional Siglo XXI. IMSS. ${ }^{4}$ Department of Pathology. Instituto Nacional de Ciencias Medicas y Nutricion Salvador Zubiran 4 . Mexico City, Mexico.

\section{References}

1. Gupta TKD, Brasfield RD, Strong EW, Hajdu SI. Benign solitary schwannomas (neurilemomas). Cancer. 1969;24:355-66.

2. Oberman HA, Abell MR. Neurogenous neoplasms of the mediastiumn. 1960;13:882-98.

3. Strollo DC, Rosado de Christenson ML, Jett JR. Primary mediastinal tumors: part II. tumors of the middle and posterior mediastinum. 1997;112:1344-357.

4. Kirschbaum A, Ritz R, Bartsch D. Giant intrathoracic left-sided vagal schwannoma. Thoracic Cardiovascular Surg Reports. 2013;2:19-22.

5. Wu Y, Zhang J, Chai Y. Giant mediastinal schwannoma located in the lower right side of the chest. Nigerian J Clin Pract. 2016;19:678-80.

6. Quartey B, Lenert J, Deb SJ, Henry LR. Giant posterior mediastinal ancient schwannoma requiring thoracoabdominal resection: a case report and literature review. World J Oncol. 2011;2:191-94.

7. García-Toral R, Aguilar-Padilla L, Gardoqui-Zurita F, Prieto-Murguía E, Vásquez-Fernández F. Giant mediastinal neurilemoma. A case report. Rev Med Inst Mex Seguro Soc. 2009;47:327-30.

8. Albores Zuñiga O, Dominguez Parra LM, Jimenez Fuentes E, Ramirez Bollas J, Ramirez Barrantes M. Schwannomas mediastinales. Comucación de casos y revisión de la literarura. Rev Inst Nac Enf Resp Mex. 2004;17:256-59.

9. Antonescu CR, Perry A, Woodruff. Schwannoma (including variants) in Fletcher CDM, et al. WHO Classification of tumours of soft tissue and bone. Fletcher CDM, Bridge JA, Hogendoom CW, Mertens Fredrik. Eds International Agency for Reasearch on Cancer. Lyon 2013.

10. Yue Y, Xin H, Xu BC, Zhang LN, Zhao W. Posterior mediastinal neurilemmoma accompanied by intrapulmonary sequestration in the left lower lobe. A case report. Medicine (Baltimore). 2019;98(30):e16582.

11. Zhu W, Chen D. Vagus nerve schwannoma in the right upper mediastinum. Thorac Cancer. 2017;8(6):698-702.

12. Song YJ, Seol SH, Kim S, Kim DK, Kim KH, Ki, DII, Kang DK, Kim JY. Benign posterior mediastinal schwannoma -multiple diagnostic imaging modalities. Clin Case Rep. 2019;7(12):2585-87.

13. Cohwn LM, Schwartz AM, Rockoff SD. Benign schwannomas: pathologic basis for CT inhomogeneities. Am J Radiol. 1996;147:141-43. 
14. Rammos KSt, Rammos SK, N Foroulis ChN, Zaramboukas Schwannoma of the vagus nerve, a rare middle mediastinal neurogenic tumor: case report. J Cardiothorac Surg. 2009; 4: 68.

15. Nakazono T, White CS, Yamasaki F, Yamaguchi K, Egashira R, Irie H, Kudo S. MRI findings of mediastinal neurogenic tumors. AJR Am J Roentgenol. 2011;197:W643-52.

16. Martinez Esteve A, Alvarez Perez RM, Pachon Garrudo VM, Gonzalez Mancha R, Borrego Dorado I. Mediastinal schwannoma mimicking lymph node metastasis from locally advanced breast in a $18 \mathrm{~F}-$ FDG PET/CT study. Rev Esp Med Nucl Imagen Mol. 2016;35:272-73.

17. Woodruff JM, Godwin TA, Erlandson RA, Susin M, Martini N. Cellular schwannoma: a variety of schwannoma sometimes mistaken for a malignant tumor. Am J Surg Pathol. 1981;5:733-44.

18. Lodding P, Kindblom LG, Angervall L, Stenman G. Cellular schwannoma: a clinicopathologic study of 29 cases. Virchows Arch A Pathol Anat Histopathol. 1990;41:237-48.

19. White W, Shiu MH, Rosenblum MK, Erlandson RA, Woodruff JM. Cellular schwannoma: a clinicopathologic study of 57 patients and 58 tumors. Cancer. 1990;66:1266-75.

\section{Figures}
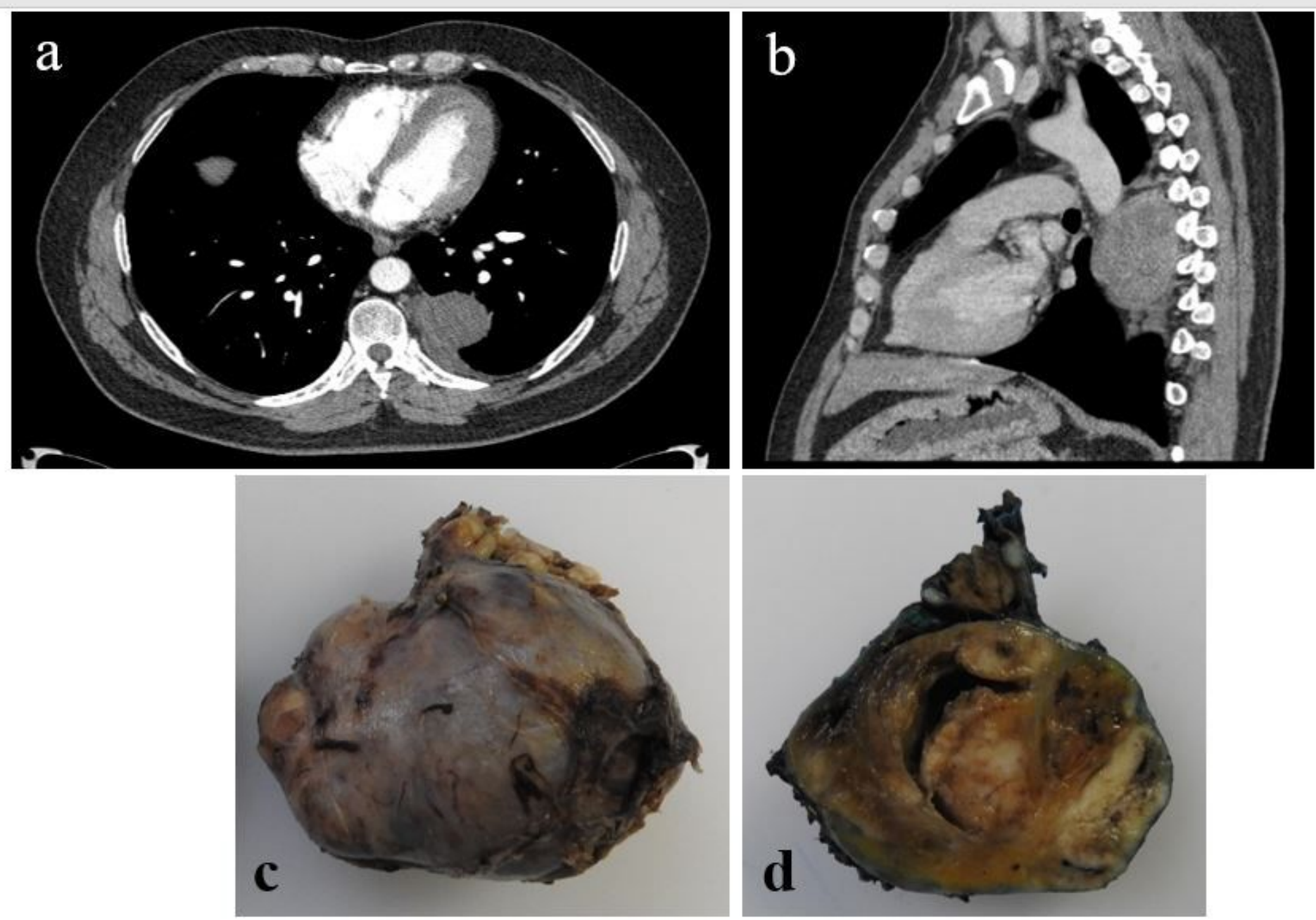


\section{Figure 1}

Benign mediastinal schwannoma. a-b) CT imagen the tumor was located in the posterior mediastinum. Tumor showed heterogeneous reinforcement with hypo and hyperintense areas after the application of contrast material; c) Macroscopic images of the well-encapsulated ovoid tumor with smooth surface, gray-yellow color; d) Cross section of the tumor shows solid areas with cystic degeneration.

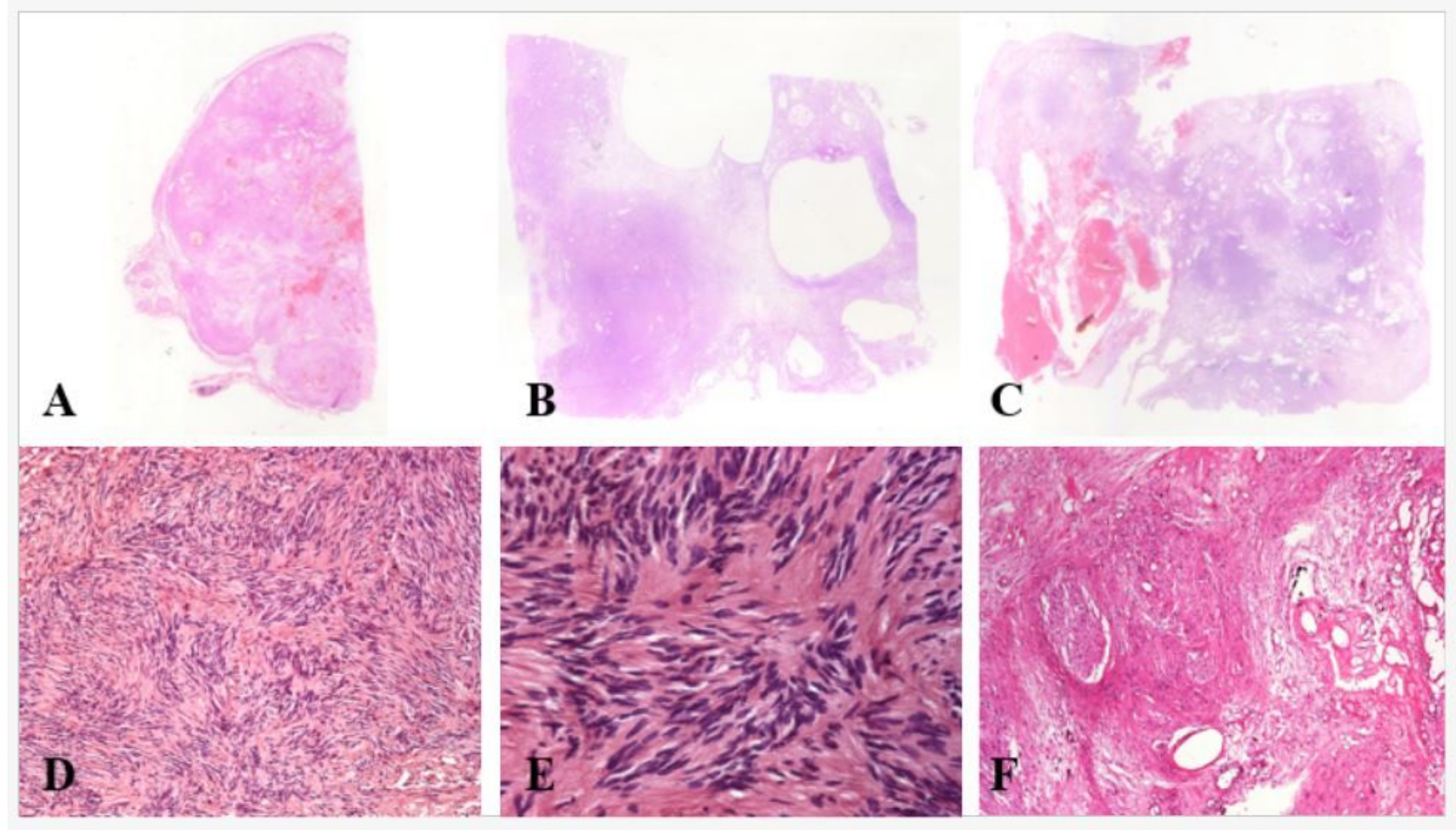

Figure 2

Microscopic images of the conventional mediastinal schwannoma. a) Encapsulated tumor with presence of nerves on the external surface; b) Solid areas with cystic degeneration; c) Areas of hemorrhage and vascular congestion; d) Antoni A and Antoni B areas with biphasic patterns; e) Verocay body, formed palisaded Schwann cells; f) Hyalinized thich-walled vassels. 

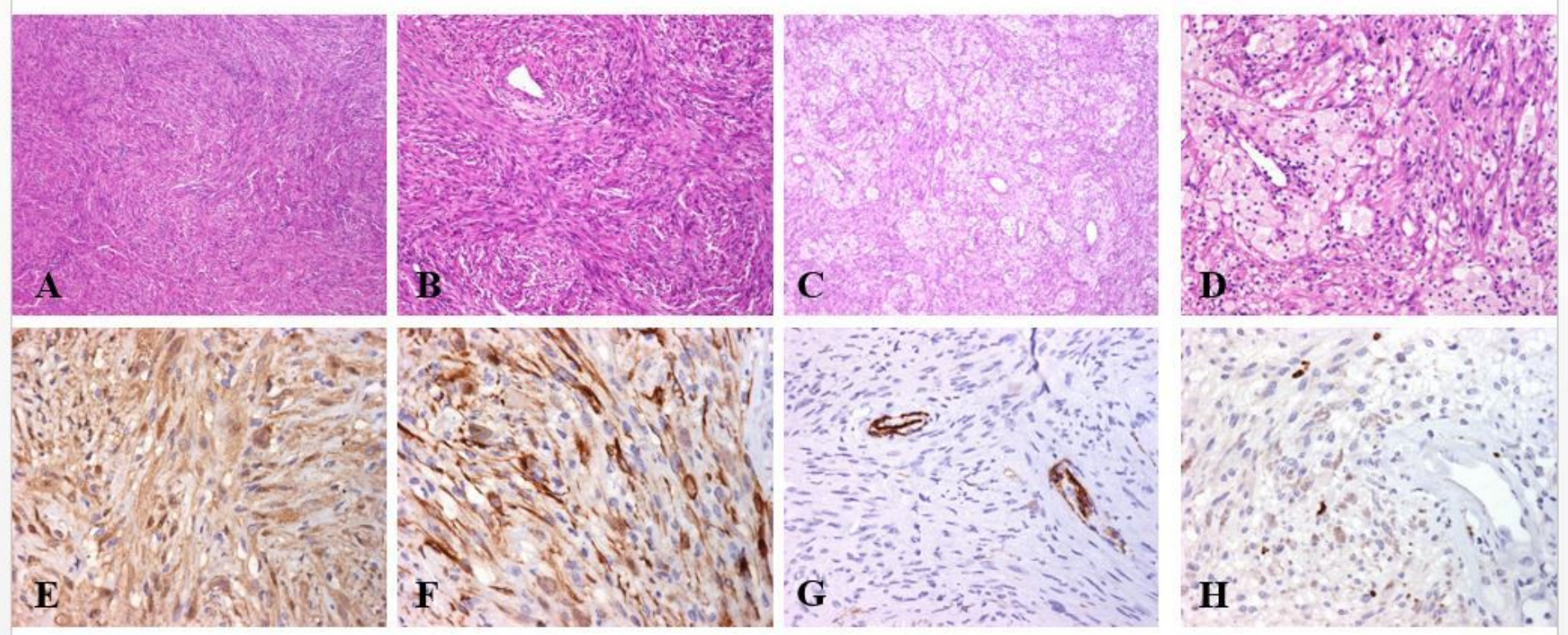

\section{Figure 3}

Microscopic images of the cellular mediastinal schwannoma. a) Tumor shows hypercellular areas; b) Cellular schwannoma with storiform growth pattern mimicking solitary fibrous tumor; c-d) Groups of numerous histiocytes; e) Diffuse expression by IHC for S100 protein in neoplastic cells; f) Intense expression by IHC for GFAP in neoplastic cells; g) Neoplastic cells were negative for CD34; $h$ ) The neoplastic cells show a proliferation index of $1 \%$ (Ki-67).

\section{Supplementary Files}

This is a list of supplementary files associated with this preprint. Click to download.

- TableS1.docx

- Tables2.docx 